Universidad de Lima

Facultad de Psicología

Carrera de Psicología

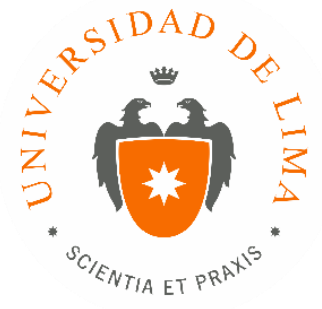

\title{
EXPERIENCIA DE TRABAJO EN UN CENTRO PSICOLÓGICO PARA NIÑOS
}

Trabajo de suficiencia profesional para optar el título profesional de Licenciado en Psicología

\section{Ariane Drago Gruslin}

Código 20122377

$$
\text { Lima - Perú }
$$

Febrero de 2019 


\section{EXPERIENCIA DE TRABAJO EN UN CENTRO PSICOLÓGICO DE NIÑOS}




\section{TABLA DE CONTENIDO}

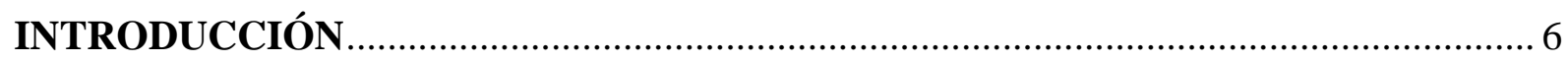

CAPÍTULO I: IDENTIFICACIÓN DEL PROBLEMA …........................................... 7

CAPÍTULO II: DESCRIPCIÓN DE LAS ACTIVIDADES REALIZADAS ................... 9

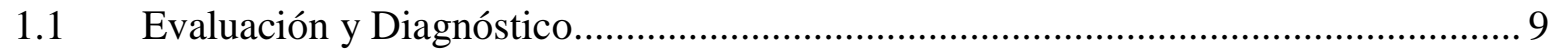

1.2 Capacitación de Acompañantes técnicos para el programa CUNA MÁS ............... 11

1.3 Programa de adaptación y acompañamiento a niños adoptados internacionales ..... 12

CAPÍTULO III: RESULTADOS DE LA INTERVENCIÓN ......................................... 14

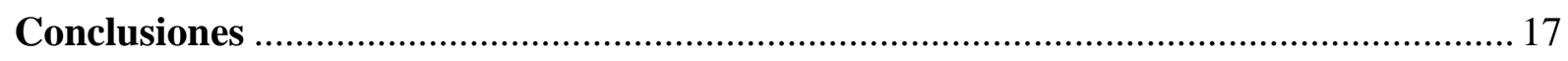

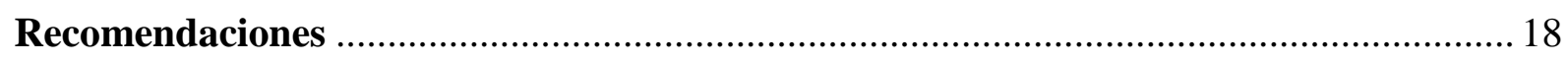

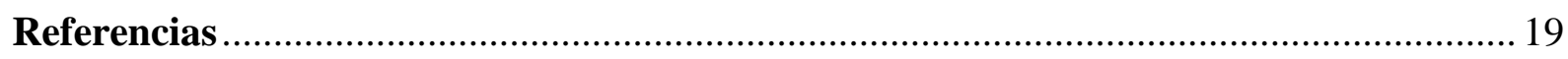

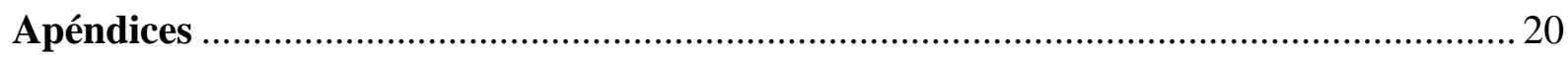




\section{ÍNDICE DE APÉNDICES}

Apéndice 1: Desarrollo de la sesión de las capacitaciones a cuna más ................................. 21

Apéndice 2: Ficha de entrevista de motivo de consulta...................................................... 25

Apéndice 3: Modelo de informe de evaluación diagnóstica .................................................... 28 


\section{INTRODUCCIÓN}

La infancia es la etapa en la cual se adquieren, mediante el aprendizaje, una gran cantidad de competencias que permitirán un desarrollo saludable en el niño (Luciano, 1997). Es en esta etapa en la que se adquirirán patrones de conducta que ejercerán una profunda influencia sobre la vida de la persona (Kostelnik, Phipps, Soderman y Gregory, 2009). Por ello mismo, es una etapa importante para identificar obstáculos y problemáticas, o incluso para potenciar sus habilidades, con miras a un desarrollo más saludable.

Los problemas de salud mental en niños y adolescentes presentan una elevada incidencia en el Perú y en el mundo, sin embargo, aún no es considerada como prioridad por parte de los gobiernos. Es por ello, que las condiciones de salud mental aún no son óptimas para dicha población, generándose una gran necesidad de que los servicios de salud mental se centren en la detección temprana y la integren como parte esencial de la atención primaria de la salud (Instituto Nacional de Salud Mental Honorio Delgado- Hideyo Noguchi [INSM], 2012). Añadido a esto, diversas estadísticas sustentan la importancia de una intervención recuperativa o preventiva en el campo de la salud mental infantil, pues se ha demostrado que mientras más temprano se realice una intervención, mayor será la efectividad de esta (Ministerio de Salud del Perú [MINSA], 2018). Es así que, desde la disciplina de la psicología, se considera de suma importancia, prevenir e intervenir a tiempo en esta etapa mediante actividades como la evaluación, diagnóstico, intervención y prevención.

Bajo este panorama, el presente reporte detallará las labores profesionales realizadas, así como los resultados observados, en un centro especializado en brindar servicios relacionados a la psicología infantil. Específicamente se centrará en las actividades de evaluación y diagnóstico, capacitación a cuidadores y acompañamiento a niños adoptados. 


\section{CAPÍTULO I: IDENTIFICACIÓN DEL PROBLEMA}

Desde la segunda mitad del siglo XX, los indicadores de bienestar social y salud física de los niños y adolescentes han mejorado notablemente a nivel mundial; sin embargo, y contrariamente a lo esperado, los índices de salud mental han venido disminuyendo. (INSM, 2012). Más específicamente, en el Perú se estima que el $20 \%$ de los niños presenta alguna vulnerabilidad de salud mental que necesita intervención, mientras que, de las atenciones en centros de salud mental comunitarios, el $70 \%$ de los pacientes son niños y adolescentes (Cutipé citado en Sausa, 2018).

Es así como la psicología infantil está cobrando cada vez más fuerza a nivel global, lo que se puede observar en un incremento en la investigación sobre la misma (Caballo y Simón, 2017). Esto es una respuesta a un contexto que lo demanda cada vez más por razones tales como un aumento de la psicopatología infantil (Sánchez y González, 2014) y brechas elevadas entre los niños que requieren tratamiento y quienes realmente lo reciben (MINSA, 2018).

Esto ha hecho que se incremente la frecuencia con la cual los padres llevan a sus hijos donde profesionales de la salud mental por motivos cada vez más diversos, lo que supone un desafío para los proveedores de estos servicios pues se tiene que estar preparado para poder responder a las problemáticas específicas. Por ejemplo, actualmente acuden a consulta niños con diferentes dificultades tales como bajo rendimiento académico, dificultades sociales, dificultades emocionales, dificultades de aprendizaje y problemas familiares; así como, con diversas psicopatologías como trastorno por déficit de atención (TDAH), depresión, ansiedad, trastorno de la comunicación social, retraso global del desarrollo, entre otros.

Al tratarse de niños, la demanda proviene principalmente de los padres o del centro de estudios, lo que significa que el contexto educativo se encuentra altamente implicado, ya que 
es ahí donde se desenvuelve el niño y son las maestras y los psicólogos escolares los que suelen solicitar evaluaciones o intervenciones en base a lo observado en ellos. Por ello, tiene que haber un trabajo articulado entre la psicóloga y el colegio.

Es así como surge el Centro en el año 2013, con el propósito de brindar un espacio para atender a familias y escuelas que buscan un diagnóstico certero, soluciones integrales y el acompañamiento positivo de profesionales especializados, para que niños y adolescentes alcancen su mayor potencial. Los servicios que brinda el Centro son evaluación y tratamiento en las áreas cognitivas, socio emocionales, lenguaje, aprendizaje y habilidades sensoriales y motoras. Desde su inicio, el Centro ha presentado un crecimiento constante debido a la demanda que ha ido incrementándose año tras año. 


\section{CAPÍTULO II: DESCRIPCIÓN DE LAS ACTIVIDADES Y TAREAS REALIZADAS}

\subsection{Evaluación y Diagnóstico}

En el Centro se desarrollaban evaluaciones integrales a niños de diversas edades y con diferentes motivos de consulta. El proceso de evaluación iniciaba teniendo una entrevista con los padres para recopilar el motivo de consulta y conocer el contexto del paciente. Con dicha información se decidía el proceso a seguir y el tipo de evaluación a realizar (Esquivel, Heredia y Gómez, 2017). Dependiendo la edad, también se realizaba una entrevista al niño o una sesión de juego diagnóstico.

Luego, se llevaban a cabo seis (6) sesiones con el niño donde se le aplicaba diversas pruebas. Por ejemplo: para medir el área cognitiva, se aplicaba la Escala de Inteligencia de Wechsler para niños WPSSI IV y WISC V (Wechsler, 2011), que además de informar el grado de inteligencia general, específica las áreas cognitivas fuertes y débiles (Esquivel, Heredia y Gómez, 2017). Es importante mencionar que dicho Test no se encuentra adaptado a la población peruana. Asimismo, para medir el área socioemocional se utilizaban pruebas proyectivas como el Test de frases incompletas de Forer, el Dibujo de la Figura Humana en los niños, el Test del dibujo de la familia y el Test del dibujo libre. Estas pruebas permiten evaluar la personalidad y los conflictos del niño. Por ejemplo, el Test de dibujo de la familia examina las vivencias conflictivas del niño con relación a su familia (Maestro, 2010). Para medir el área de lecto-escritura, no se utilizaba una 
prueba estandarizada sino una lectura simple acorde a la edad del evaluado. Primero, se le dictaba la lectura al menor para que la escriba. Luego, debía leerla en voz alta para finalmente, responder a preguntas específicas sobre la lectura y de cultura general relacionadas al texto. A través de este método, se medía la caligrafía, ortografía, pronunciación, velocidad lectora y el manejo de los signos ortográficos y de puntuación, así como también, la comprensión lectora. Esta evaluación se calificaba de forma cualitativa.

Además, siempre que el caso lo requiriese, se pactaba una reunión con la profesora y la psicóloga del colegio para conocer su perspectiva del caso y adicionalmente, se observaba al niño en el salón de clase durante 45 minutos. Esto permitía conocer de primera mano el desenvolvimiento del niño a nivel social. Igualmente, se solía hacer una visita al hogar, lo cual permitía analizar el vínculo entre la madre y el hijo en el momento del juego, así como también el espacio físico donde el niño pasaba la mayor parte de su tiempo. Adicionalmente, siempre que el menor llevara alguna terapia en el centro, se pactaba una reunión con la terapista para obtener información adicional.

Finalmente, después de haberse obtenido los datos suficientes a través de las diferentes técnicas de evaluación utilizadas, el caso se analizaba con un conjunto de especialistas, con quienes se llegaba a un diagnóstico y se ideaba el plan de tratamiento adecuado y acorde al diagnóstico (Maestro, 2010). Se preparaba un informe que constaba de las diversas áreas analizadas, él diagnóstico presuntivo, basado en las clasificaciones psiquiátricas pertenecientes al Manual Diagnóstico y Estadístico de los Trastornos Mentales - DSM-5 (Asociación Americana de Psiquiatría, 2013) y las recomendaciones. Posterior a ello, el proceso evaluativo concluía con la de devolución de los resultados a los padres. En caso fuera 
necesario, y con el fin de psico educar a los padres y así facilitarles la comprensión de la información brindada, en ocasiones se armaba una presentación donde se explicaba el diagnóstico y los criterios cumplidos por el menor. Por último, en caso fuera pertinente se pactaba una reunión de devolución de resultados y recomendaciones con el centro de estudios.

\subsection{Capacitación de Acompañantes técnicos para el programa CUNA MÁS}

Otra de las actividades realizadas en el Centro fue brindar capacitación a los Acompañantes Técnicos de Cuna Más (en adelante, los “A.T”) acerca de la regulación de emociones, comportamientos y resolución de conflictos en niños y niñas menores de 3 años. Cuna Más es un programa social estatal a cargo de Ministerio de Desarrollo e Inclusión Social cuyo objetivo es mejorar el desarrollo infantil de niños menores de 3 años en zonas de pobreza para que logren superar brechas propias de la pobreza en su desarrollo cognitivo, social, físico y emocional (Ministerio de Desarrollo e Inclusión Social, 2017-2018). Las capacitaciones fueron dictadas por una antropóloga, una psicóloga y una practicante de psicología. La modalidad de estas capacitaciones era "formador de formadores" ya que los A.T luego iban a instruir a las madres cuidadoras, responsables de cuidar a los niños en las salas de cuidado diurno. El objetivo del taller era fortalecer sus capacidades en el manejo de estrategias para la regulación emocional de los menores. Dichos talleres se realizaron en Cusco, Arequipa y Lima. Por cada ciudad se realizaron 2 talleres, cada uno de 4 horas. Para realizar los talleres previamente se diseñaron diversos productos tales como diapositivas con sustento teórico, dinámicas vivenciales, separatas, dípticos y una guía para que los A.T puedan replicar la 
capacitación. Luego de finalizar la capacitación en cada ciudad se realizaba un informe y se presentó el trabajo realizado con la representante adjunta de UNICEF, ya que UNICEF trabaja directamente los programas del ministerio de inclusión social y cuna más es uno de ellos.

\subsection{Programa de adaptación y acompañamiento a niños adoptados}

\section{internacionales}

En el Centro se realizó un programa para trabajar con dos niños adoptados de Ucrania. Estos niños son hermanos de 8 y 10 años. El objetivo del programa fue insertarlos en el nuevo sistema escolar y familiar. Según Berástegui (2007), son diversos los estudios que consideran que los niños adoptados, tanto nacionales como internacionales, tienen riesgo de presentar problemas de adaptación sociofamiliar, afectiva, y conductual. Sin embargo, según el estudio realizado por Berástegui, los adoptados internacionales tienen mayor riesgo de desarrollar problemas de salud mental.

Uno de los puntos relevantes que refirieron los padres, fue que ellos no esperaban que los niños tuvieran problemas de adaptación, pues pensaban que los niños se iban a adaptar fácilmente e iban a estar agradecidos por haber sido adoptados. Sin embargo, contrario a lo que esperaban, los ninos se mostraron frustrados y presentaron conductas agresivas frente a la dificultad de adaptarse a una nueva cultura y a un nuevo entorno familiar.

El trabajo que se hizo fue progresivo y gradual. Este trabajo inició por visitas diarias al hogar luego de que los menores llevaran dos semanas viviendo en Lima, y se dio durante un mes. El objetivo fue generar un vínculo de confianza con ellos, a través de actividades lúdicas y manualidades. El trabajo se realizó en el idioma 
inglés ya que los menores no hablaban castellano. Adicional a ello, se les brindaba retroalimentación diaria a los padres y se les sugería los ajustes necesarios a nivel de crianza, como también a nivel del vínculo madre-hijo y respecto a las reglas del hogar para los menores.

Asimismo, al contemplar que los menores no tenían los niveles educativos ni emocionales esperados para ingresar al colegio se optó por escolarizarlos durante el primer año en el Centro con la compañía de 2 profesoras y de la psicóloga, quien formaba parte de su círculo de confianza. La psicóloga actuaba de mediadora y los calmaba ante la frustración y el miedo, entre otras emociones intensas que experimentaban. Adicionalmente, tanto en la fase 1 (trabajo en casa) como en la fase 2 (escolarización en el Centro), la psicóloga encargada tomaba notas del comportamiento diario y estas eran notificadas a la coordinadora del programa, para hacer los ajustes necesarios, y a los padres, a quienes se les brindaba la retroalimentación correspondiente. Luego de 2 meses de escolarización, cuando los menores ya estaban adaptados a asistir diariamente al centro, la psicóloga comenzó a retirarse progresivamente, hasta que los menores ya pudieron permanecer solos con las profesoras y, a la par, iniciar un proceso terapéutico. 


\section{CAPÍTULO III: RESULTADOS DE LA INTERVENCIÓN}

Con respecto a la primera función, evaluación y diagnóstico, se aportó satisfaciendo la demanda de diversos padres y centros educativos. Se contribuyó mediante la aplicación y corrección de instrumentos psicológicos, así como en la elaboración de los informes y la realización de presunciones diagnósticas, las cuales permitían generar una mayor comprensión sobre las problemáticas de los niños y sus orígenes. Esto, además de brindar un sentido a los padres sobre los signos y síntomas observados en los niños, permitía idear un plan de tratamiento completo y acorde a la problemática para que los menores alcancen un desenvolvimiento óptimo en diferentes áreas.

Estos resultados se evidenciaron mediante el agradecimiento de los padres, luego de realizar la devolución de los resultados, las recomendaciones y la propuesta de intervención. Asimismo, se evidenció buenos resultados mediante la continua demanda por parte de los colegios y sus palabras positivas hacia el trabajo realizado. Finalmente, también se observó la presencia o la asistencia de nuevos pacientes que llegaban al centro a manera de recomendación por parte de los padres que ya habían hecho uso de los servicios previamente. En cuanto a la realización de capacitaciones para los A.T de Cuna Más, estas tenían como principal objetivo mejorar las condiciones de cuidado hacia los menores. En primer lugar, se capacitó a 272 A.T en el uso de herramientas efectivas de cuidado para que estos los repliquen en las madres cuidadoras y, a su vez, estas replanteen los métodos de atención a los niños que estaban en la sala de cuidado diurno. También, se trabajó la importancia de que el A.T pueda brindar apoyo emocional hacia las madres cuidadoras, quienes solían presentar altos niveles de estrés y agotamiento, lo cual influye directamente en su trabajo diario con los niños. Puesto que, si las madres cuidadoras se encontraban emocionalmente indispuestas por 
sus dificultades personales, esto podría afectar la calidad del cuidado y trato hacia los niños bajo su responsabilidad.

Los resultados de dicha intervención se evidenciaron mediante el agradecimiento de los A.T's, los cuales tenían comentarios positivos hacia las expositoras y se reflejaron en las evaluaciones de salida resueltas por los participantes. Asimismo, se evidenció la satisfacción del contratante mediante las continuas invitaciones para seguir capacitando en diferentes provincias. También, al culminar las capacitaciones y observar los resultados, UNICEF decidió nombrar a la directora del Centro como asesora oficial de la institución.

En cuanto al programa de adaptación y acompañamiento a niños adoptados, se logró desarrollar un vínculo de confianza entre los niños y la psicóloga, con el que se logró que los menores expresaran sus vivencias internas y los miedos que tenían con relación a su adopción. Este vínculo permitió que los niños accedan a recibir una educación personalizada y una mejora en la adaptación hacia el medio familiar, lo cual se observó en una mejor comunicación con los padres, aceptación de su nuevo hogar/ país y en la reducción de las conductas impulsivas. Finalmente, se dio una buena evolución que fue evidente gracias al reporte de los padres, ya que frecuentemente mencionaban los avances que observaban en los menores, que, si bien aún presentaban ciertas conductas impulsivas, estas se reducían cada vez más. Asimismo, las profesoras encargadas mencionaban la importancia del trabajo realizado con ellos previo a la escolarización personalizada. Es importante mencionar que los menores habían sido aceptados en un colegio, pero que por decisión de todas las partes involucradas se decidió escolarizarlos de manera personalizada reservando la vacante para el próximo año. En tal sentido, los avances que venían presentando los menores frente al plan de tratamiento eran reportados hacia el colegio, con el fin involucrar a la institución en el proceso, para que el siguiente año los menores puedan hacer una transición adecuada a su nuevo centro de estudios. El colegio tenia comentarios positivos hacia el trabajo gradual y 
progresivo que se había realizado con los menores. Asimismo, es importante mencionar que dicho colegio comenzó a recomendar a diversas familias el Centro. 


\section{CONCLUSIONES}

- El proceso de evaluación y diagnóstico es fundamental para conocer a mayor profundidad las problemáticas de los niños y así plantear un plan de tratamiento acorde a sus necesidades específicas.

- La evaluación diagnóstica permite a la psicóloga trabajar en conjunto con el centro de estudios, armando un plan de tratamiento articulado entre el centro psicológico y el centro de estudios, buscando así potenciar los recursos del menor en el aula.

- Es importante aplicar diversas pruebas y no solo una, ya que mediante la triangulación entre ellas aumenta la validez de los resultados.

- Respecto a la adaptación de niños adoptados internacionales, se considera de suma importancia trabajar con un plan estructurado y gradual, respetando la individualidad del menor y sin forzar a que se desarrolle al mismo nivel que los niños de su edad.

- Con respecto a la realización de capacitaciones a los A.T, se concluye que es importante capacitar a las personas que se dedican al cuidado de niños, pero también es importante que se les brinde un espacio donde puedan ser contenidas y escuchadas, ya que en la medida que se sientan bien brindarán un mejor cuidado a los menores. 


\section{RECOMENDACIONES}

A continuación, se detallarán las recomendaciones:

- Se le sugirió al Centro en múltiples ocasiones utilizar pruebas validadas en el país para medir el coeficiente intelectual en los niños. Sin embargo, estas sugerencias no fueron consideradas y debido a que uno se rige bajo las normas de la institución, en este caso, el Centro (donde se realizó el internado), se tuvo que aplicar dicha batería de pruebas utilizada por el centro de trabajo.

- Es importante utilizar pruebas estandarizadas para medir el nivel de lecto-escritura en los niños. Se sugirió medir dicha área con pruebas psicológicas estandarizadas, pero finalmente se siguió evaluando bajo la modalidad del Centro. Asimismo, se le hizo notar al centro que las preguntas de cultura general presentaban una dificultad elevada para la edad de los niños evaluados.

- Se recomienda que los padres que vayan a tomar la decisión de adoptar a niños internacionales de una edad en la que tienen uso de razón, deben prepararse con un especialista en el tema debido a que los niños adoptados no solamente tienen que adaptarse a un nuevo entorno familiar sino a un nuevo país.

- Se le recomienda al programa Cuna Más, capacitar directamente a las madres cuidadoras pues ellas son las que encargadas de cuidar a los niños para que superen las brechas que los afectan debido a la pobreza en la que viven.

- Las capacitaciones a los A.T de Cuna Más deberían ser más de una vez al año para que se mantengan actualizados y no dejen de brindar el soporte necesario a las madres cuidadoras. Sin embargo, como el presupuesto es limitado se podrían trabajar asesorías por teléfono o por mensajería telefónica con la finalidad de resolver dudas que pueden surgir en la aplicación de las técnicas aprendidas. 


\section{REFERENCIAS}

Asociación Americana de Psiquiatría. (2013). Guía de consulta de los criterios Diagnósticos del DSM-5. Arlington,VA: Asociación Americana de Psiquiatría.

Berástegui, A. (2007). La adaptación familiar en adopción internacional: un proceso de estrés y afrontamiento. Anuario de Psicología, 38(2), 209-224. Recuperado de https://www.redalyc.org/pdf/970/97017404004.pdf

Caballo, V. E., y Simón, M. A. (2017). Manual de psicología clínica infantil y del adolescente. Trastornos Específicos. Madrid: Ediciones Pirámide

Esquivel, F., Heredia, M. A., y Gómez, M. L. (2017). Psicodiagnóstico clínico del niño. México D.F.: El Manual Moderno.

Instituto Nacional de Salud Mental "Honorio Delgado - Hideyo Noguchi”. (2012). Estudio epidemiológico de salud mental de niños y adolescentes en Lima metropolitana y callao 2007. Anales de Salud Mental, 18(1). Recuperado de http://www.insm.gob.pe/investigacion/archivos/estudios/2007-ASM-EESM-NYA.pdf

Kostelnik, M. (2009). El desarrollo social de los niños. México D.F.: Cengage Learning.

Luciano, C. (1997). Características del comportamiento en la infancia y la adolescencia. En Luciano, C. (Ed), Manual de psicología clínica. Infancia y adolescencia (pp. 21-70). Valencia: Promolibro.

Maestro, C. (2010). Uso práctico de la evaluación psicológico. En C. Soutullo y M. J. Mardomingo (Eds.), Manual de psiquiatría del niño y del adolescente (pp. 25-35). Pamplona: Editorial Médica Panamericana.

Ministerio de Desarrollo e Inclusión Social. (2017-2018). Programa Nacional Cuna Más. Lima: Programa Nacional Cuna Más. Recuperado de http://www.cunamas.gob.pe/

Ministerio de Salud del Perú. (2018). Plan nacional de fortalecimiento de servicios de salud mental comunitaria 2018-2021. Recuperado de http://bvs.minsa.gob.pe/local/MINSA/4422.pdf

Sánchez, P., y Valentín, O. (2014). Urgencias en psiquiatría infantil. Pediatría Integral, 18(5). Recuperado de https://www.pediatriaintegral.es/publicacion-201406/urgencias-en-psiquiatria-infantil/

Sausa, M. (3 de febrero de 2018). Menores ocupan el 70\% de atenciones en salud mental [infografía]. Perú 21. Recuperado de https://peru21.pe/peru/cifras-salud-mental-perumenores-ocupan-70-atenciones-infografia-394376

Wechsler, D. (2011). Escala de Inteligencia de Wechsler para Niños IV (WISC-V). Buenos Aires: Paidós. 
APÉNDICES 


\section{APÉNDICE 1: DESARROLLO DE LA SESIÓN DE LAS CAPACITACIONES A CUNA MÁS}

\section{Programa Nacional \\ GU DESARROLLO DE LA SESION \\ Objetivos de la CAPACITACION}

- Hacer sentir al acompañante técnico que está siendo atendido en sus necesidades como acompañante técnico.

- Formar un vínculo entre el equipo capacitador y el A.T.

- Lograr el interés del acompañante técnico por la preocupación de la dimensión emocional de la madre cuidadora.

\section{DESARROLLO DE LA SESION:}

\section{Presentación y Bienvenida (10 minutos)}

- La persona se presenta y cuenta su experiencia laboral.

- Da a conocer la importancia y los objetivos del taller.

\section{Recojo de información (50 minutos)}

1. Se le explica la importancia de recoger información acerca de lo que ellas piensan, lo importante que es conocer el punto de vista de ellas, para ello se le comunica que has traído dos preguntas que tienen que responder y se lee las preguntas en voz alta, brindándole un ejemplo para una mayor comprensión:

¿Cuáles son las dificultades más importantes que ustedes observan en las madres cuidadores en relación al manejo emocional con los niños que están a cargo?

¿Qué habilidades piensan que requerirían las M.C desarrollar más para atender el desarrollo emocional de los niños a su cargo?

2. Se entregan papeles de 2 colores y se le pide a las Madres cuidadoras que respondan una pregunta en cada papel.

3. En grupos de 5 personas, se comparte las ideas y se llega a ideas FUERTES.

4. Se les da un papelógrafo por grupo, donde deberán apuntar las ideas en común y las ideas fuertes encontradas para ambas preguntas.

5. Se elige un representante del grupo y salen al frente a exponer el papelógrafo.

6. Uno de los capacitadores anota en la pizarra los puntos fuertes por grupo.

7. Se cierra hablando de lo encontrado: aquí uno utiliza lo que se apuntó en la pizarra, lo que más llamo la atención en cada grupo sobre las dificultades encontradas y las habilidades a desarrollar por ellas. La capacitadora, hace un breve resumen y señala 
la importancia de reconocer el desgaste emocional y el cansancio emocional que interfiere en que la madre cuidadora no sea activa, sino por el contrario sea evasiva y permisiva.

Se le explica que, como seres humanos, los mismos acompañantes técnicos tienes desgaste emocional y cansancio y que se requiere de ordenar sus emociones para poder ayudar a otros.

8. Se conecta la reflexión final con el primer tema teórico.

\section{TEORIA: (30 min): Contención ante las necesidades (lectura emocional).}

\section{Temas principales:}

$\checkmark$ Vinculo en la rutina diaria

$\checkmark$ El trato emocional para la madre cuidadora

$\checkmark$ La mochila personal

$\checkmark$ La importancia de fortalecer a la madre cuidadora

$\checkmark$ Empatía

$\checkmark$ La regulación afectiva

$\checkmark$ Estrategias para demostrar empatía

\section{BREAK (30 minutos)}

\section{Dinámica (30 min): Yo te guio, tú me guías}

La experiencia consiste en guiar a otro que va con los ojos cerrados por un viaje imaginario donde hay retos y peligros, y también espacios apacibles y tranquilos de mayor libertad.

Se guía con el contacto, la sonoridad y la palabra.

Luego se comparte la experiencia para vincularla con el tema la confianza, la vulnerabilidad, el cuidado y los límites.

\section{- Instrucciones para realizar la dinámica:}

1. Vamos a hacer una ronda, todos hagan un circulo grande.

2. Le voy a dar a cada uno un número, presenten atención porque necesito que se lo acuerden.

3. La capacitadora le asigna el número "1" y el número "2" de manera intercalada a cada persona de la ronda.

4. Se les comunica a todos que los numero "1" formen una fila a la mano derecha y el numero " 2 " formen otra fila a la mano izquierda. 
5. Se le explica que los números "1" son los guía y los numero "2" son los guiados. Por lo tanto, el número "2" cierra los ojos y el numero "1" le da instrucciones al número dos para avanzar como, por ejemplo: "camina de frente, agáchate, levanta la mano, detente". Se dice "comiencen" hasta que diga "Deténganse".

6. Cuando se da la consigna para que paren, se les dice que se coloquen en fila, por un lado, todos los numero "1" y por otros los numero "2".

7. Se le entrega un papelógrafo a cada grupo y se le pide que cuenten que sintieron, como se sintieron al ser guías y en el otro grupo como se sintieron al ser guiados (a este último se le indica que ponga información respecto al tono de voz de su guía, las palabras que uso para guiarlo y el contacto corporal, si este era tosco o delicado)

8. Luego exponen en grupo las ideas escritas en el papelógrafo. Permaneciendo un grupo frente al otro.

9. La capacitadora vincula la experiencia con la confianza, la vulnerabilidad, el cuidado y los límites, que se da en la relación Asistente técnica- madre cuidadora.

\section{Dinámica (40 minutos): Recuerda y reflexiona una experiencia de buen trato y de mal trato.}

La experiencia consiste en recordar algún momento de la niñez de ser acogido y nutrido, o de ser abandonado o maltratado.

Reconocer en parejas y en grupo que tienen en común estas experiencias y cuales han sido sus resultados y consecuencias.

Recoger las ideas del grupo en relación al tema del trato, las emociones y el estrés. Vincular con lo que hacen ellas mismas y las Madres Cuidadoras.

\section{Instrucciones:}

1. Cierra los ojos, relájense, respiren, suelten el cuerpo, gira la cabeza en círculos. Ahora: Recuerda una anécdota en tu niñez en que sentiste que no le importabas a alguien, quien estaba, donde estabas.. (2 minutos en silencio).

2. Abre tus ojos y escribe la experiencia en un pedazo de papel.

3. Dibuja lo que sentiste a través de un trazo. Por ejemplo: si sentiste cólera, realiza un trazo fuerte. Si sentiste incomodidad un trazo sutil.

4. Cierra los ojos nuevamente. Ahora: Recuerda una anécdota en tu niñez en que sentiste que si le importabas a alguien ( 2 minutos en silencio).

5. Abre tus ojos y escribe la experiencia.

6. Dibuja lo que sentiste a través de un trazo.

7. Ahora escoge una pareja, una persona que este al costado tuyo y siéntense mirándose frente a frente y compartan: que cosas nos hacen sentir pocos importantes para los demás y que cosas te hacen sentir importante. 
8. Luego se invita a las personas voluntariamente a contar como se sintieron, si les gusto sentirse escuchadas a no.

9. La capacitadora realiza una reflexión de la importancia de sentirse escuchadas y de tener una red de apoyo.

\section{TEORIA: (30 min): Estrés y el efecto domino en el trato}

\section{Temas principales:}

$\checkmark$ Autorregulación emocional

$\checkmark$ Reacciones del estrés (cólera, angustia, miedo)

$\checkmark$ Afrontamiento del estrés

$\checkmark$ Evasión del estrés

$\checkmark$ Consecuencias del estrés en la madre cuidadora

$\checkmark$ Signos de estrés en la madre cuidadora

$\checkmark$ ¿Por qué es importante saber reconocer el estrés en las madres cuidadoras?

$\checkmark$ ¿Qué ocurre con el niño cuando la madre cuidadora esta estresada?

$\checkmark$ Estrés genera limitación

\section{TEORIA (30 minutos): Trato con ternura y límites}

\section{Temas principales:}

$\checkmark$ CO Regulación del adulto para el logro de la autorregulación del niño

$\checkmark$ Explicarle el concepto de Límite: que el niño identifique el peligro y que es saludable para él, para su desarrollo. " aquí en la pared no, pero en el papel si "

$\checkmark \quad 0$ castigo. Porque cerebralmente no calza dentro de un niño de $0^{\prime} 3$ meses. Si le enseñas el castigo, malogras el vínculo, su autoestima, miedo, angustia y esto se va a repercudir en los diferentes ámbitos. Configurando una química cerebral de angustia.

$\checkmark$ Necesita amor, cariño.

$\checkmark$ Herramientas para desarrollar la noción de autoridad en la rutina diaria

$\checkmark$ Herramientas para marcar limites

\section{PREGUNTAS Y CIERRE (10 minutos)}

- Se agradece y se invita a hacer las ultimas preguntas o dudas que hayan quedado

- Se entrega la separata/ díptico con información

- Se entrega y se le pide que llenan la encuesta de satisfacción 


\section{APÉNDICE 2: FICHA DE ENTREVISTA DE MOTIVO DE CONSULTA}

\section{Historia personal de nuestro hijo}

\section{Datos personales del niño}

Nombres y apellidos:

Edad:

Fecha de nacimiento:

Colegio/nido, grado:

¿Hace cuánto tiempo está en este colegio?

\section{Motivo de consulta}

Razón por la cual buscan ayuda psicológica y/o psicopedagógica:

Si resumiéramos en pocas palabras, que es lo que más preocupa en su hijo(a):

¿Cuáles son las expectativas respecto a esta ayuda?:

¿Quién los refirió?:

¿Cómo es la relación actual entre los padres? (descríbala):

Como son ustedes como padres con su hijo(a), explique minuciosamente cada uno por separado:

\section{Datos de los hermanos}

¿Cuántos hijos tiene la pareja:

Mencione el nombre y edad de cada uno de los hijos:

¿Cómo se lleva su hijo(a) con cada uno de los hermanos y qué comparte con cada uno?(describa):

\section{Datos de la dinámica familiar}

- ¿Quiénes forman parte de la familia? (especifique):

- ¿Qué otras personas viven en la casa?

- ¿Cómo es la dinámica familiar en un día de semana?

- ¿Cómo son los sábados?

- ¿Cómo son los domingos?

- ¿Viajan?, ¿cómo son los viajes?, ¿con qué frecuencia?

- ¿Qué hacen usualmente en vacaciones?

- ¿Qué tipo de problemas y preocupaciones tiene la familia?

\section{$\underline{\text { Su hijo(a) }}$}

Describa cómo es su hijo(a), lo más detallado que pueda:

¿Cuáles son sus fortalezas?

¿Cuáles son las cosas que tiene que mejorar?

¿Qué es lo más que le preocupa en su hijo(a)?

¿Qué le gusta hacer?

¿Qué es lo que no le gusta hacer?

¿Con quién se lleva mejor en la casa?

Vida social

¿Qué tan sociable es?

¿Se ve con los amigos con frecuencia?, ¿cuándo?, ¿Dónde?

¿De dónde provienen sus amigos?

¿Qué actividades realiza con los amigos?

Estudios 
Cómo le va en los estudios:

En qué cursos va mejor:

En que cursos no le va bien:

Tiene o ha tenido ayudas de profesores, cuáles, cuánto tiempo, que resultado dieron:

Tiene algún problema específico en sus aprendizajes (descríbalo):

Ha tenido alguna repitencia (explique):

Ha tenido algún cambio de colegio (explique):

\section{Otras actividades}

Qué hace en sus tiempos libres:

A qué le gusta jugar:

Con quién juega:

Realiza algún deporte:

Qué otra actividad realiza usualmente:

Tiene algún hobbie:

\section{Alimentación}

Cómo es su alimentación (describa):.

Come comida chatarra:

Tiene algún problema con el peso:

\section{Sueño}

Cómo duerme (describa): Tiene o ha tenido dificultades con el sueño (explique):

Tiene o ha tenido temores nocturnos (explique):

Cuántas horas duerme regularmente:

\section{Temores}

Tiene temores, a qué:

Alguien más en la familia tiene temores, cuáles, cómo los manejan:

\section{Impresiones}

Ha tenido impresiones fuertes, cuál o cuáles, cuándo (explique):

Cómo las manejaron, ha quedado algún efecto posterior:

\section{Métodos formativos - correctivos}

Qué acciones realiza la familia para formar temas de disciplina, obediencia, respeto y similares:

Qué métodos usan para corregir alguna acción que no se desea o acepta:

Cómo reacciona su hijo: aprende.

\section{Salud}

Ha tenido algún problema de salud antes de importancia, o tratamiento, intervención quirúrgica, accidente o similar:

Alguna vez ha tenido consulta con un neurólogo o psiquiatra (explique):

Tiene problemas de aguda visual o auditiva:

Tiene o ha tenido algún tratamiento psicológico (explique y especifique cuándo inició y cuándo duró y qué logros se tuvieron):

Tiene o ha tenido alguna terapia (explique y especifique cuándo inició y cuándo duró y qué logros se tuvieron):

\section{Embarazo}

Explique si hay algún dato importante a tomar en cuenta: 


\section{Parto}

¿Cómo fue?, ¿hubo alguna dificultad? (explique detalladamente):

¿Cuál fue el puntaje del Apgar?

\section{Primer año de vida}

Explique temas importantes en relación, al sueño, lactancia, alimentación, desarrollo motor, lenguaje: Desarrollo motor:

lenguaje:

\section{Control de esfínteres:}

Explique temas importantes en relación al control, edad, recaídas:

\section{Algún otro tema importante a tomar en cuenta:}




\title{
APÉNDICE 3: MODELO DE INFORME DE EVALUACIÓN DIAGNÓSTICA
}

NOMBRE Y APELLIDOS DEL

EVALUADO

\section{INFORME PSICOLOGICO}

\author{
DATOS GENERALES \\ $X X X$ es una nilla do $X$ ahos $y \mathrm{X}$ mes que acbualmenbe se encuentra cursando $X X X$ \\ grado en el cologio XXX. \\ MOTNO DE CON SULTA \\ HISTORIA DE DESARROLLOY CONTEXTO FAMILIAR
}

TECNICAS UTILIZADAS

PROCESO EVALUATMO

AREA COGNITINA

En el indice de Razonamianto Fluldo.

Con respecto al indice Viroespeolal,

En nelación al indice de Mamorla de Trabajo (MT)

El indice do Comprention Varbal.

Finaimento, en el indice do Valooldad de Prooscamianto,

EVALUACION DE LO3 PROCE3O3 DE LECTURA Y E3CRITURA

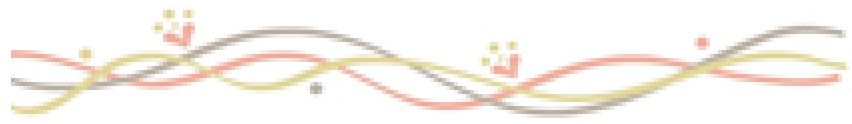


AREA EMOCIONAL

ANALISIS DE RESULTADOS

RECOMENDACIONES

Lima de do

I 